\title{
Different performance, full experience: a learning game applied throughout adulthood
} \author{
Simon Greipl ${ }^{1}$, Korbinian Moeller ${ }_{4664-8430]}^{1,2,4,5,6}$, Kristian Kiili ${ }^{3}$, Manuel Ninaus ${ }^{1,4[0000-0002-}$ \\ ${ }^{1}$ Leibniz-Institut für Wissensmedien, Tübingen, Germany \\ \{s.greipl,k.moeller, m.ninaus\}@iwm-tuebingen.de \\ ${ }^{2}$ Department of Psychology, Eberhard-Karls University, Tuebingen, Germany \\ ${ }^{3}$ TUT Game Lab, Tampere University of Technology, Pori, Finland \\ kristian.kiili@tut.fi \\ ${ }^{4}$ LEAD Graduate School \& Research Network, University of Tuebingen, Germany \\ ${ }^{5}$ Centre for Mathematical Cognition, School of Science, Loughborough University, \\ Loughborough, United Kingdom. \\ ${ }^{6}$ Individual Development and Adaptive Education Center, Frankfurt am Main, Germany
}

\begin{abstract}
Gaming may not primarily be associated with the elderly part of the population, but senior gamers represent a growing proportion. Evaluating the applicability of educational games across the lifespan is therefore crucial to better understand potential requirements in different domains and age groups. The current study applied a math learning game to participants from 19 to 79 years of age. We examined age-related differences along three domains: cognition, as measured by in-game performance, basic player experience with the game, and general player attitudes towards technology. Analyses of performance metrics related to speed clearly reflected an age-related decline, well in line with known effects from cognitive ageing. In contrast, errormetrics were associated with educational background. At the player experience level, however, we found no differences across the lifespan. All participants rated the game equally (positive). Furthermore, our results showed that attitudes towards technology only differed occasionally, for instance, senior participants seemed to be more technologically interested. Taken together, scoring systems as well as other in-game metrics based on performance, especially related to speed, should be adapted appropriately to player's age. Finally, elderly players' experience with the game and overall attitudes towards technology appeared comparable to other younger age groups.
\end{abstract}

Keywords: game-based learning, life-long learning, applicability, user-experience, elderly, number line estimation;

\section{Introduction}

Video gaming is an established and popular mainstream activity among teenagers and (young) adults (e.g. [1]). However, there is also a substantial number of gamers aged 50 and above. The Entertainment Software association (ESA), for instance, indicates that $21 \%$ of gamers are over 50 years of age and thereby constitute the age group - together with the 
age group of individuals of 18 years and younger - with the second highest percentage of gamers [2]. According to Huizinga's proposition of a 'Homo Ludens' [3], there seems to be no decline in the engagement in playful activities with age. This leads to the interesting question why the attempt to utilize, for instance, the motivational potential of educational games, still focuses mainly on younger populations (e.g. [4]). Educational research widely acknowledges that play is a central and natural way for children to learn and acquire new skills [5]. Yet, there seems to be a traditional dichotomy between learning and playing [6] in the sense that learning and playing are often separated in formal and especially higher education. However, the gradual detachment of learning and playing in secondary and tertiary education [7] seems paradox [8].

In the present study, we employed a math game used for the assessment and training of fraction magnitude understanding [9], [10], which was so far primarily played by primary and/or secondary school students. As topic fractions were chosen because they are considered to be one of most difficult relations to learn in math education and even adults frequently fail to process them correctly ([11], [12] for a review). Therefore, we applied the game to age groups from 19 to 79 to examine differences of game-related performance as well as subjective experiences with respect to educational games and attitudes towards technology in general. In the following, we first provide some general information on gaming in (older) adults, followed by background on three domains we deemed relevant when investigating cross-age effects in the current study, namely (i) cognition, (ii) player experience, and (iii) player attitudes.

\subsection{Related work}

\subsubsection{Games and their applications for the adult and elderly population}

In general, games used in rehabilitation or for rehabilitation purposes are becoming increasingly popular to foster motivation of patients [13] and can be distinguished in three types addressing physical, cognitive, and social aspects [14]. Games targeting physical and cognitive effects make up most of the studied effects. Importantly, about $75 \%$ of studies reported in a review by Ngyuen and colleagues [14] also found a positive impact on wellbeing in the elderly. However, there are restrictions in the interpretation of these results. From originally over 6000 articles only 24 were finally retained for synthesis and the authors critically noted that more emphasis should be put on research designs. Most of the reviewed games were utilized to compensate impairments and disabilities on the physical or cognitive level (e.g., memory, attention, problem solving etc.), acquired through diseases or injury, and to overcome repetitive characteristics of therapy and training processes [15]. So far, most articles examined the impact of digital games on well-being, brain plasticity or decreasing cognitive abilities in (children and) seniors. Outside the rehabilitation context, however, healthy adult populations were found to benefit from game-based trainings as tools to enhance cognitive and emotional skills as well [16]. Importantly, the use of gamebased training and learning across all age groups is not only a highly relevant topic [17], but also provides new challenges that need to be overcome by researchers and game designers. In particular, the use of games for the elderly goes beyond aspects of compensation of age-related or incidence-related declines and therefore requires a framework for game-based lifelong learning [6] or intergenerational game-design workshops [18] to promote, for instance, intergenerational learning and exchange. Accordingly, there is a need for better understanding the requirements, needs, as well preferences of elderly people when using game-based (learning) applications.

\subsubsection{Gaming across age}

The gerontoludic manifesto [19] advocates (amongst others) that gaming research and design needs to focus on heterogeneity rather than stereotyping. That is, older gamers vary greatly in terms of preferences, experiences, or health status, and these differential factors should be accommodated for in game design. Not only for the adult, but also for the 
(healthy) elderly population, this raises the question whether commonly used games in the context of learning or even entertainment maintain their applicability with respect to their main qualities, they are usually evaluated on. While mobile applications were already successfully used to assess cognitive functions across the lifespan e.g. [20], to our knowledge only one study examined the same gaming environment on heterogeneous age samples. By following such an approach, this means, keeping the gaming environment the same, confounds by different gaming environments can be controlled for allowing a clear focus on individual variables. In this single study, the authors assessed motor development with a Kinect sensor across the lifespan embedded in a serious game for rehabilitation [21]. The authors replicated that cognitive performance across the lifespan first increases and then decreases again - a quadratic trend known from neuropsychological evidence on maturation [22] and aging [23].

To advance and extend this knowledge to the cognitive domain in terms of acceptance and applicability of serious games, we used a tablet-based math game that was primarily played by and designed for non-adult populations. According to previous research, the acceptance of educational games should be independent of gender, technical expertise, gaming frequency, and only weakly influenced by age. It seems that the acceptance of games is more influenced by the perceived fun or positive game experience, respectively [24]. That is, subjective experiences should have a high impact on the acceptance of educational games. In contrast, performance in the game should mostly be influenced by age and previous gaming experience [24]. For the current purpose, we followed a similar distinction, separately evaluating the game with respect to the domains Cognition and (subjective) Player Experiences/Attitudes.

\subsubsection{Player cognition \& (cognitive) ageing}

Besides actual clinical conditions, there is a natural association between aging and a decline in cognitive and perceptual abilities as well as an increase in physical impairments which should be considered in the design of games (e.g. [25], [26]). Age related cognitive decline is most significantly found in components of fluid cognition [27] such as processing speed [28], whereas the crystallized part of cognition, that is knowledge and experience, is comparably stable, rarely starting to decline until the age of 65 [27]. A general view on the cognitive performance trajectory describes the development across ages as a quadratic trend with a short plateau of maintaining cognitive functioning around the mid 30's [29]. This quadratic relationship was replicated by a serious game for rehabilitation applied across the lifespan [21]. However, crystallized parts of intelligence that depend on learning and represent experience may even rise until the age of 70 [30] (Figure 1). Fluid parts of cognitive functioning indicate "...the ability to identify complex relations and to draw inferences on the basis of that comprehension..." [29, p. 132] and are thought to reach their climax around the mid 20's (see also Figure 1). According to the authors, the quadratic trend of cognitive performance is, amongst others, a realistic, interactive view on fluid and crystallized systems of intelligence that also implies that crystallized capacities may also decline with age as unused knowledge representations may be forgotten over time [29].

Another relevant factor is age-related decline in physical capabilities. Deteriorations in this domain are often indicated by, for instance, grip strength, chair rising ability, walking speed, time to get up and standing balance performance [31]. These can be reduced down to three motor factors balance (e.g., single chair stand), fine motor skills (e.g., put water from one hand to the other) and flexibility (touch left earlobe with right hand behind the head, and vice versa) [32]. Handling difficulties among elderly players have been reported on several occasions, for instance with balancing boards (Nintendo Wii console) or the sensitivity of Wii controllers (Wiimote) being too high [33], meaning that at least balancing and fine motor skills may be important to consider. In summary, physical engagement in games can be modulated by a decline in motor abilities, which could lead, for instance, to difficulties with the handling of the device (e.g., using sensitive gyroscopic 
controls like twisting or tilting the device) or for controlling the game and its mechanics (e.g., cascaded inputs with high complexity and precision).

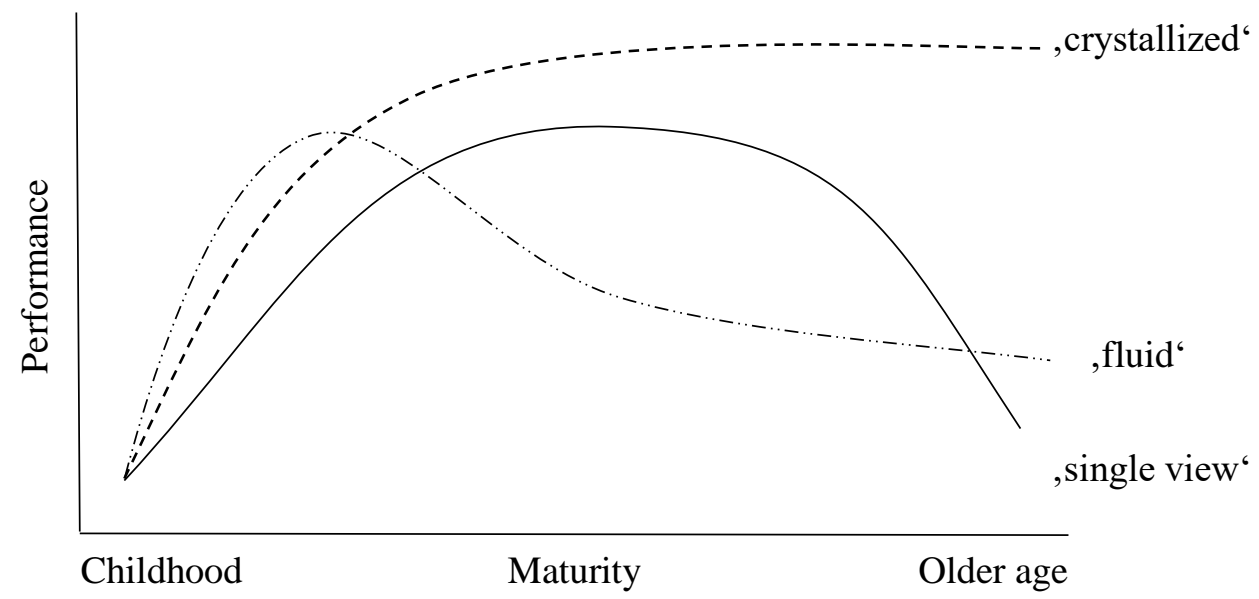

Figure 1. Cognitive ageing (theoretical): the 'single view' indicates interaction between fluid and crystallized systems of intelligence including the more realistic view that representational knowledge can also be lost in higher ages. Adapted from [29].

\subsubsection{Player experiences}

We indicated earlier that the experiential domain like perceived fun majorly impacts on the acceptance of educational games [24]. Thus, whether and how players across different age groups might experience an (educational) game differently seems of critical interest not only for acceptance, but potentially also for interactions with in-game performance. Accordingly, flow - a widely used measure in game-based learning [34] - might be a construct desirable to be assessed in this context. Flow is generally considered a positive emotional state (e.g. [35], [36]) and reflects a cognitive-experiential factor that describes the balance between demands of the game and player's resources. This balance may be different for people of different ages or outside a given target group. Most educational games are not adaptive to the player and are mostly designed to fit a very specific target group. As flow experience measures the fluency and automaticity of cognitions during gaming, it may eventually be taken as a presupposition for successful learning and a positive gaming experience.

Moreover, other, more general aspects of user experience [37] such as attractiveness, pragmatic quality (e.g., handling), and hedonic quality (e.g., stimulation) of a game might be of particular relevance when investigating cross-age effects. These general aspects of user experience are often investigated when evaluating software (e.g. [37]) and can provide subjective experiences related to the mere interaction with a game-based learning environment. In contrast to flow measures, that, for instance, give hints on how focused users were, these subjective user experience scales reflect, amongst others, the overall look and feel of the game. Furthermore, aspects of pragmatic quality (e.g., ease of handling the game) might be particularly important when comparing younger to older players.

\subsubsection{Player attitudes}

Evaluating the applicability of (educational) games across a broad age range requires considering the players' (educational) background and socialization. While these factors are inherently important when evaluating (educational) games, they might be even more important in a sample covering a broad age spectrum. To evaluate educational games, usually more targeted and homogenous samples like secondary school students are used. Compared to that, our sample is probably much more heterogeneous. This becomes for instance apparent because elderly gamers seem to play different games than younger ones. 
For instance, they often prefer card and puzzle games like solitaire and scrabble [2] over, for instance, action games and first person shooters. It may be that such preferences reflect socialization and prior experience, this means todays senior gamers did not grow up with digital games but analogue games, because digital games were not available at that time. However, their preferred games also comprise "digitalised" pendants of classic games, for instance puzzle games like scrabble. However, literature indicates that gaming habits are less indicative for the acceptance of a game [24]. In contrast, there still is a gap present today in the use of media between younger and older generations. This age-related differential use of technology is best evaluated with respect to internet use and has been described as the digital or grey divide (see e.g. [39]-[41]). It refers to the observation that media use is much more widespread among younger generations compared to the elderly part of the population. This divide may also inherent some attitudinal differences towards media and the use of technology. For instance, a rare use of technology might be due to a lack of interest in these technologies of a person. But the very same person may also be highly interested with just a lack of opportunity or access. Accordingly, such attitudes may have an impact on the perception and therefore the applicability of our learning game.

Overall, we would not expect the elderly population to have a very different mindset on "playing games" or the usefulness of games or playing in general. However, different perspectives on (the use of) technology are of interest especially in senior gamers. Therefore, the current study investigated relevant attitudinal aspects beyond mere cognitive and experiential factors. At first, we assessed attitudes towards technology regarding a personal and a societal level with respect to the two dimensions: learning and working as well as entertainment and communication. Second, we evaluated attitudinal differences towards the specific electronic device (a tablet-PC) the game is played on. This should provide additional information on whether there are relevant handling issues specifically attributed to the device the game is played on. Third and last, we addressed the question whether there is an age-related change in general affinity and attitude towards technology [42], [43], which in the context of serious games has not been evaluated so far.

\section{The present study}

The educational game used in the current study was developed to assess and train fraction magnitude understanding. Generally, deficits in such basic numerical competencies have critical drawbacks not only on a developmental but also societal level [44]. It is therefore crucial to establish and maintain numerical competencies across age. The game used in the current study comprises two tasks: (i) The number-line estimation task in this game requires the player to navigate an avatar along a horizontal number-line to accurately indicate the correct location of a target fraction (e.g., where goes $4 / 7$ on a number line from $0-1$ ?). This type of task is commonly used for training number magnitude understanding [e.g., 44] and performance in this task is associated with mathematical achievement [46]. (ii) The magnitude comparison task simply requires deciding which one of two fractions is the larger (or smaller) one. Both tasks can be evaluated in terms of speed (i.e., how long it takes to solve a comparison/number-line estimation) and error rate (i.e., how many incorrect items per session). The number-line estimation task also allows for assessing the accuracy of a given estimate (i.e., how close was the indicated solution to the correct location).

The overall objective of the current study was to investigate the applicability of educational games across age. More specifically, we investigated three potential domains relevant when using (educational) games across a broad age spectrum, namely cognition, (subjective) player experience, and attitudes: 


\section{Player cognition}

Age related cognitive declines are most significantly found in components of fluid cognition [27] such as processing speed [28], whereas the crystallized part of cognition, this means knowledge and experience, is comparably stable, rarely starting to decline until the age of 65 [27]. While in-game performance measure such as speed can clearly be attributed to fluid cognition, error rates and accuracy should be more (fraction) knowledge-dependent and therefore part of crystalized cognition in adult individuals. Therefore, we expected an overall age-related decline in performance. However, this should be much more prominent for in-game metrics associated with speed, but not necessarily for others such as estimation accuracy. We further hypothesised that, besides age-related differences, some in-game performance metrics might be influenced by participants' prior tablet use and their gaming frequency. Specifically, some user problems may arise due to the usage of the tablet-PC when playing the game. As speed metrics may primarily show age-related differences, these might also be in the focus of possible or even mandatory adaptions necessary to make games age appropriate. It is, for instance, feasible, that a speed-related scoring system could make it unreasonably hard for an elder player to get achievements or reach the next level in the game.

\section{Player experiences}

General experience when playing an educational game is another important domain when investigating potential differences across age as these might influence users' adoption in the future or hint towards potential changes in game design. Therefore, we investigated the state of flow and general user experience during playing the educational game used in the current study [24]. However, based on previous research one would not expect large age-related differences on these variables if the educational game is designed appropriately. Hence, when handling the device (e.g. tilting the tablet) or the game itself (learning the game mechanics) is not problematic, we would not expect significant differences between the age groups on flow and general user experience.

\section{Player attitudes}

Elder people use technology and games differently, which may be rooted in different habits and/or attitudes towards technology and gaming. It is conceivable that, for instance, a strong negative position towards technology may hinder the acceptance of educational games. Accordingly, we completed our investigation on the applicability of an educational game across age by evaluating central parts of these attitudinal aspects. That is, general attitudes towards the use of technology and relevant attributions on a personal and societal level, usefulness of tablets, as well as general affinity towards electronic devices might differ across age.

\section{Methods}

The game took about 10-15 minutes to complete and involved both number line estimations and magnitude comparisons. For both tasks we compared speed and the number of correctly solved items across age. Additionally, we compared accuracy of number-line estimation across age. For the experiential and attitude related part of the analysis, we divided the participants into three age groups (see section 2.1 below). Data presented in this paper are part of a larger project including other measures such as general cognitive abilities as well as an assessment of basic math competencies, which are not relevant for the current study. 


\subsection{Participants}

141 adults from three age groups participated in the current study: (i) 51 participants below 35 years of age ( $M=24.61$ years; $S D=3.62$ years; 37 females), (ii) 47 participants between 35 to 59 years of age ( $M=46.13$ years; $S D=7.42$ years; 29 females) and (iii) 43 participants aged 60 years and above ( $M=66.72$ years; $S D=3.91$ years; 20 females). Participants were recruited via online and newspaper advertisements. The study was approved by the local ethics committee. Age of participants ranged from 19 to 79 years of age. Because prior experience with gaming and gaming habits are of critical interest, we depicted the relationship between gaming frequency and age $[r(139)=-.031, p<.001]$ in Figure 2.

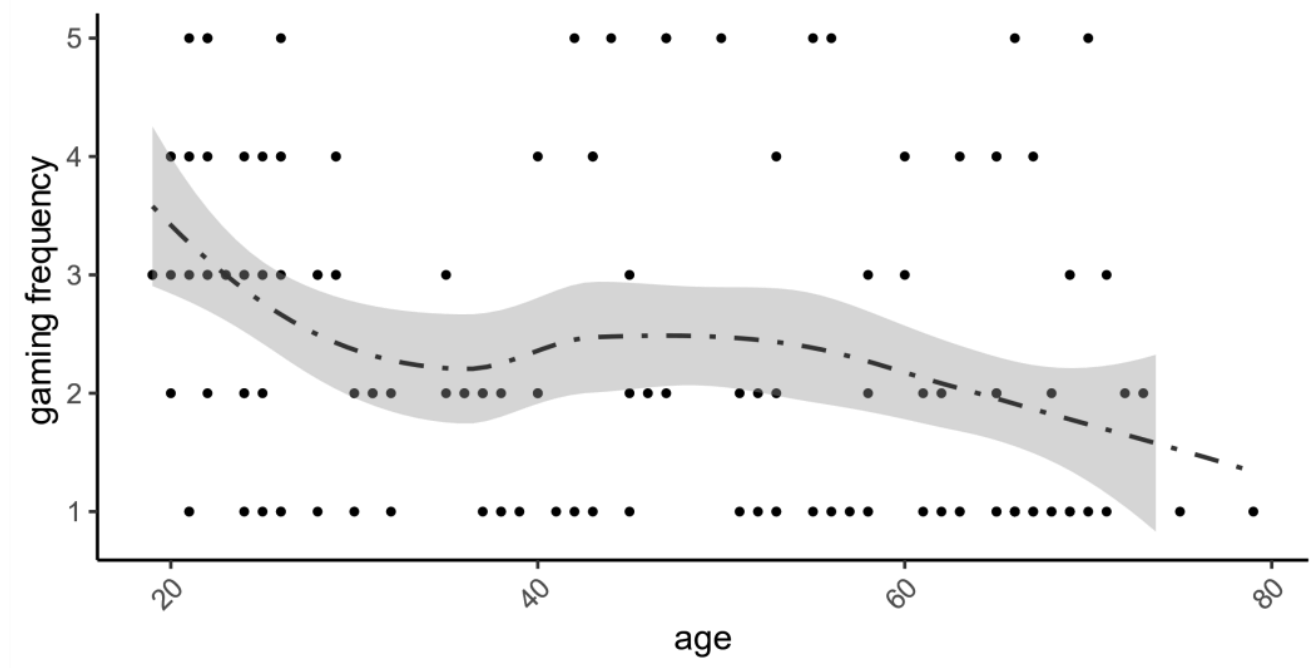

Figure 2. Distribution of our sample's gaming frequency by age measured from $1=$ never to $5=$ daily. Dashed line represents a smoothed regression line.

\subsection{Measurements}

The first and main part of the current study was the assessment with our educational game Semideus. As mentioned above, the game consisted of two tasks, number-line estimation and magnitude comparison. In the former, participants were asked to indicate the spatial position of a target number/fraction (e.g. 5/8) along a number-line with fixed end points (e.g., 0 and 1; see Figure 3). This task was complemented by the comparison task, in which participants had to put two fractions, represented by two piled blocks that displayed two different fractions, in ascending order with regard to the numerical magnitudes depicted on them (e.g., which is larger 4/7 or 1/2; see Figure 3).

The game was played on an Apple iPad. The main mechanics of the game required controlling the avatar walking along the number-line by tilting the tablet. All other operations such as confirming to be at the right position upon the number line were realized via button presses on top-left or top-right positions on the touchscreen. The game-session involved an onboarding phase and the actual assessment phase. The former was assisted by the experimenter and aimed at familiarizing players with controls and game mechanics. Therefore, whole numbers instead of fractions were used in this phase. The latter was played without assistance and only these data were analysed in the current study (for further details see also [9] and Figure 3 and https://youtu.be/rhl88VvGCvI).

As such, assessments regarding the cognitive domain of players took place in-game. The time to solve each item (speed), the number of correctly solved items (error rate), and for number line estimation the accuracy of correct estimations was used (note that an estimation was considered correct when it was not more than $8 \%$ away from the correct location). We assume that these three measures can be attributed to different aspects of intelligence. The time to solve each item (speed) should be clearly related to fluid parts of intelligence, whereas the number of correctly solved items (error rate) may be reliant on prior 
training/exercise with fractions (e.g. represented by the educational level of the participant). Estimation errors seem therefore to be representative of the crystallized parts of intelligence. The accuracy of correct estimations may finally be indicative for both aspects of intelligence, because estimations generally need prior fraction knowledge (crystallized) as well as a initial learning phase and adaptation to the control, handling and use of the game (fluid).

As part of the scoring system of the game, players received coins as a function of their performance. In the estimation task, the number of coins is dependent on estimation accuracy ( $>98 \%$ correct 500 coins; $95 \%-98 \%=300$ coins; $92 \%-94 \%=100$ coins). In the comparison task, coins were awarded based on response time (under $9 \mathrm{~s}=500$ coins; 9 - 12 $\mathrm{s}=400$ coins; $12-16 \mathrm{~s}=300$ coins; $16-20 \mathrm{~s}=200$ coins; over $20 \mathrm{~s}=100$ coins; wrong answer $=0$ coins and 20 units of virtual energy loss). After completing all levels, the coinscore of each level is summed and the remaining energy is transformed into coins (energy $\% * 500$ coins) and added up as a bonus reward. This resulting overall score of the game is used as dependent variable in a later analysis step.

The second part of the current study comprised questionnaires regarding the (subjective) experiences and the attitudinal domain. To measure subjective experiences with the game we employed the short flow scale (FKS [47]). It assesses flow on two subscales on a likert scale from 1 (does not apply) to 7 (is true): automaticity/fluency of actions (e.g., "I have no difficulty concentrating") and absorption (e.g., "I do not notice time passing"). Furthermore, we used the User Experience Questionnaire (UEQ [37]). With bipolar ratings from 1 to 7, it evaluates conventional usability aspects (i.e., efficiency, e.g., "quick vs. slow"; perspicuity, e.g., "complicated - simple"; dependability, e.g., "predictable - unpredictable"), user experience (i.e., novelty, e.g., "creative - unimaginative"; stimulation, e.g.: "boring - thrilling"), and attractiveness (e.g. "pleasant - unpleasant"). These measures are combined to three scales, namely pragmatic quality (perspicuity, efficiency, dependability), which reflects goal-directed aspects, hedonic quality (stimulation, novelty), which indicates non goal-directed aspects, and lastly attractiveness as a pure valence dimension.

Table 1. Details of the subscales of the FIDEC Questionnaire with translated examples from [48]

\begin{tabular}{|c|c|c|c|}
\hline \multicolumn{3}{|c|}{ dichotomous dimensions of FIDEC (INCOBI-R) } & example items \\
\hline \multirow{4}{*}{$\begin{array}{l}\text { personal } \\
\text { experience } \\
\quad(\mathrm{PE})\end{array}$} & \multirow{2}{*}{$\begin{array}{l}\text { learning and } \\
\text { working (LW) }\end{array}$} & $\begin{array}{c}\text { beneficial } \\
\text { tool/useful } \\
\text { technology }(\mathrm{BT})\end{array}$ & $\begin{array}{c}\text { A computer is a useful tool for my } \\
\text { work. }\end{array}$ \\
\hline & & $\begin{array}{l}\text { autonomous } \\
\text { entity/uncontrollable } \\
\text { technology }(\mathrm{AE})\end{array}$ & $\begin{array}{l}\text { To me, the computer seems too } \\
\text { unreliable to use as a learning tool. }\end{array}$ \\
\hline & \multirow{2}{*}{$\begin{array}{l}\text { entertainment } \\
\text { and } \\
\text { communication } \\
\text { (EC) }\end{array}$} & BT & $\begin{array}{l}\text { The computer is an important } \\
\text { entertainment medium in my life. }\end{array}$ \\
\hline & & $\mathrm{AE}$ & $\begin{array}{c}\text { I believe that the Internet is poorly } \\
\text { organized and difficult to } \\
\text { understand. }\end{array}$ \\
\hline \multirow{4}{*}{$\begin{array}{l}\text { consequences } \\
\text { for society } \\
\text { (CS) }\end{array}$} & \multirow{2}{*}{ LW } & BT & $\begin{array}{l}\text { Computers can be very useful in the } \\
\text { teaching delivery of some subjects. }\end{array}$ \\
\hline & & $\mathrm{AE}$ & $\begin{array}{l}\text { Computer technology destroys } \\
\text { more jobs than it creates. }\end{array}$ \\
\hline & \multirow[t]{2}{*}{$\mathrm{EC}$} & BT & $\begin{array}{l}\text { Understanding between people } \\
\text { from different cultures will improve } \\
\text { because of the growth of computer } \\
\text { networks. }\end{array}$ \\
\hline & & $\mathrm{AE}$ & $\begin{array}{l}\text { Due to electronic communication, } \\
\text { human relations are and will remain } \\
\text { more superficial. }\end{array}$ \\
\hline
\end{tabular}


Additionally, to assess relevant attitudinal constructs we used items from the FIDEC questionnaire of the German revised version of the computer literacy inventory (INCOBIR) [49], which measures computer-related attitudes. The FIDEC questionnaire differentiates along the lines of potential kinds of computer use (learning and working vs. entertainment and communication), evaluations referring to people's attribution to computer technology (personal experience vs. consequences for society), and a valence rating in terms of negative or positive beliefs about the computer (beneficial tool or useful technology vs. autonomous entity or uncontrollable technology) [48]. These 3 dichotomous distinctions result in a $2 \times 2 \times 2$ combination and a total of 8 bipolar scales $(-2=$ don't agree to $0=$ neutral to $+2=$ agree). For examples of single scales please see Table 1 .

To avoid overstraining of participants filling out questionnaires, in particular older participants, we decided to use only a subset of items for each scale of the FIDEC questionnaire. Nevertheless, all constructs were at least measured by two items.

Furthermore, we employed subscales from the technology usage inventory (TUI) [50]. TUI comprises attitudinal scales allowing to evaluate specific devices as well as scales indicating general attitudes towards technology. For the current purposes, we used three of the eight scales of TUI (user friendliness, e.g. "The use of Tablet PCs is complicated" and usefulness, e.g., "A Tablet PC would help me to do my daily tasks more comfortably.") by specifically relating them to evaluate tablet-PCs. Additionally, we used one scale that indicates general interest in technology and to what extent a person tries to stay informed about new technological developments (interest, e.g., "I inform myself about technological developments."). TUI items were measured on a Likert scale from $1=$ not at all to $7=$ entirely true.

Next, we assessed a rather broad construct of positive/negative attitudes towards technology, or in this case "electronic devices", with the questionnaire regarding general affinity for technology [Fragebogen zur Technikaffinität (TA-EG) [51]). It assesses negative/positive attitude towards the use of electronic devices with 19 items and requires participants to respond to statements concerning technology use on a Likert scale from $1=$ not at all to 5 = entirely true (positive example: "Electronic devices enable a high standard of living.", negative example: "Electronic devices make ill.")

Finally, gaming frequency ( $5=$ never to $1=$ daily; scale was recoded for analysis), tablet use ( $1=$ never to $7=$ daily $)$ and educational background $(1=$ secondary school (basic high school diploma) to $5=$ academic degree) were assessed on a Likert scale.
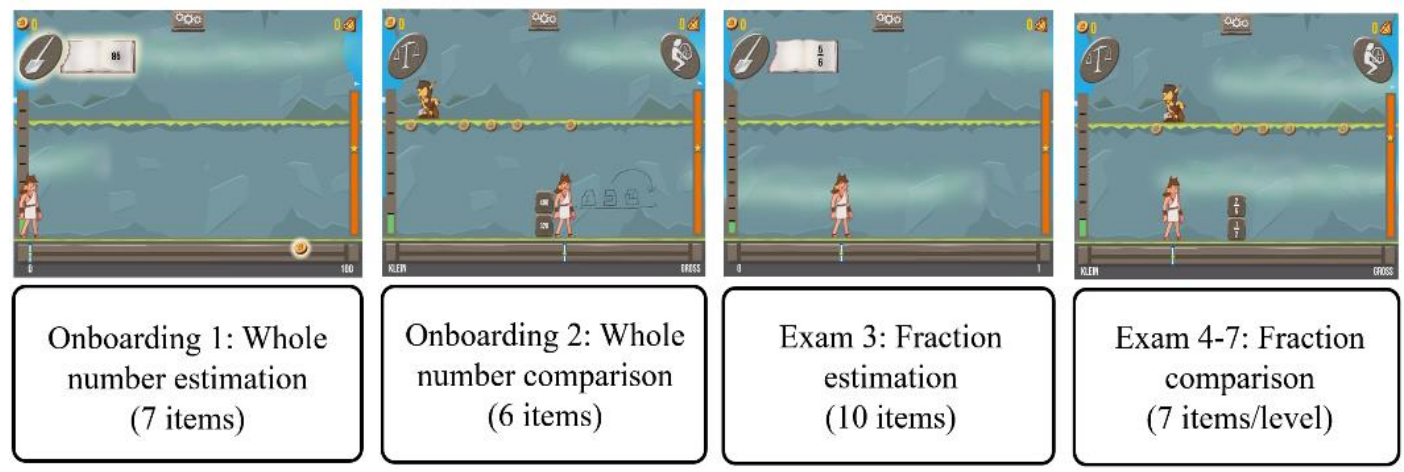

Figure 3. Screenshots of "Semideus" showing the level structure.

\subsection{Procedure}

Before playing the game on the tablet participants received written instruction on the handling and the tasks within the game. Then, participants started the onboarding phase which included 7 number-line estimation tasks and 6 magnitude comparison tasks, both with whole numbers instead of fractions to lower the initial hurdle. The actual assessment phase using fraction contained one level with 10 estimation items and three comparison levels with 7 items each. Gameplay was immediately followed by the utilized questionnaires. 
pag. 70

\subsection{Analyses}

Cognition: To evaluate how age contributes to explaining variability of in-game performance, we initially established a simple regression model including age as the only predictor. In that preliminary step, we used the overall score achieved in the game as dependent variable representing a combined measure of all established variables such as accuracy or speed while solving the game-based tasks. We compared this model to our full multiple regression model that included the four predictors age, gaming frequency, tablet use frequency, and educational level to examine potential improvement of the initial model. We then proceeded using the best model to establish the remaining five models of interest, namely those predicting speed and number of errors separated for both the comparison and the number line estimation task, as well as estimation accuracy in the number-line estimation task. In addition to that, we compared all models to their quadratic equivalents to evaluate possible quadratic relationships known from cognitive ageing.

Player experience and attitudes: To investigate potential age-related differences, in the domains of player experience and attitudes as assessed by questionnaires, we divided participants in three age groups (1: 29 - 34 years, 2: $35-59,3: 60$ and above, see section 2.1 ) and used separate multivariate analysis of variance (MANOVA). For more detailed follow-up analyses, all analyses of the experiential and attitudinal domains were complemented by post-hoc $t$-tests with FDR-correction [52] for multiple comparisons.

\section{Results}

\subsection{Player Cognition}

Our initial full model using the overall score (i.e., coins \& bonus coins earned throughout the game) as dependent variable and all four predictors (i.e., age, gaming frequency, tablet-use, education) was significant $\left[R^{2}=.28, F(4,136)=13.43, p<.001\right]$. The simple model with, again, the overall score as dependent variable but only age as a predictor was significant as well $\left[R^{2}=.15, F(1,139)=24.99, p<.001\right]$. However, the full model with all four predictors was superior compared to the simple model as it explained a significantly larger part of variance $\left[\Delta R^{2}=.13, F(3,139)=8.27, p<.001\right]$. Inspection of the beta weights indicated that age predicted the overall score significantly in the simple model $\{b=-13.91$, $95 \%$ CI $[-19.42,-8.41], t(139)=-5.00, p<.001\}$ as well as in the full model $\{b=-11.45,95 \%$ CI $[-16.86,-6.04], t(136)=-4.18, p<.001\}$. Apart from that, gaming frequency $\{b=120.07$, 95\% CI $[48.61,191.54], t(136)=3.32, p=.001\}$ and educational level $\{b=189.40,95 \%$ CI $[85.24,293.57], t(136)=3.60, p<.001\}$ predicted the overall score significantly in the full model, whereas tablet usage frequency did not. Finally, the quadratic model did not show a significant improvement in variance explained compared to the linear model $\left[R^{2}=.29\right.$, $\left.F(5,135)=10.85, p<.001 ; \Delta R^{2}<.01, F(1,136)=0.10, p=.750\right]$. Therefore, we went on using the full model in the more specific analyses. To evaluate players' cognition in more detail as measured by in-game performance variables, we proceeded our analysis using separate regression models for each in-game variable with our full model including all four predictors (i.e., age, gaming frequency, tablet use frequency, and education). As can be read from Table 2, we ran a total of five regression models in this step.

The first model with speed as the dependent variable in the comparison task, therefore related to aspects of fluid intelligence, was significant, with age and gaming frequency as significant predictors. Inspection of the beta weights (see Table 2) indicated that speed increased with more frequent gaming and decreased with age.

The second model with speed (fluid intelligence) as dependent variable in the number line estimation task was significant with only age contributing significantly to explained variance: increasing age was associated with slower task solving times in number line estimations. 
Table 2. Statistical details of the regression models using the full model in the domains speed, error and accuracy within the comparison tasks and the number line estimation tasks.

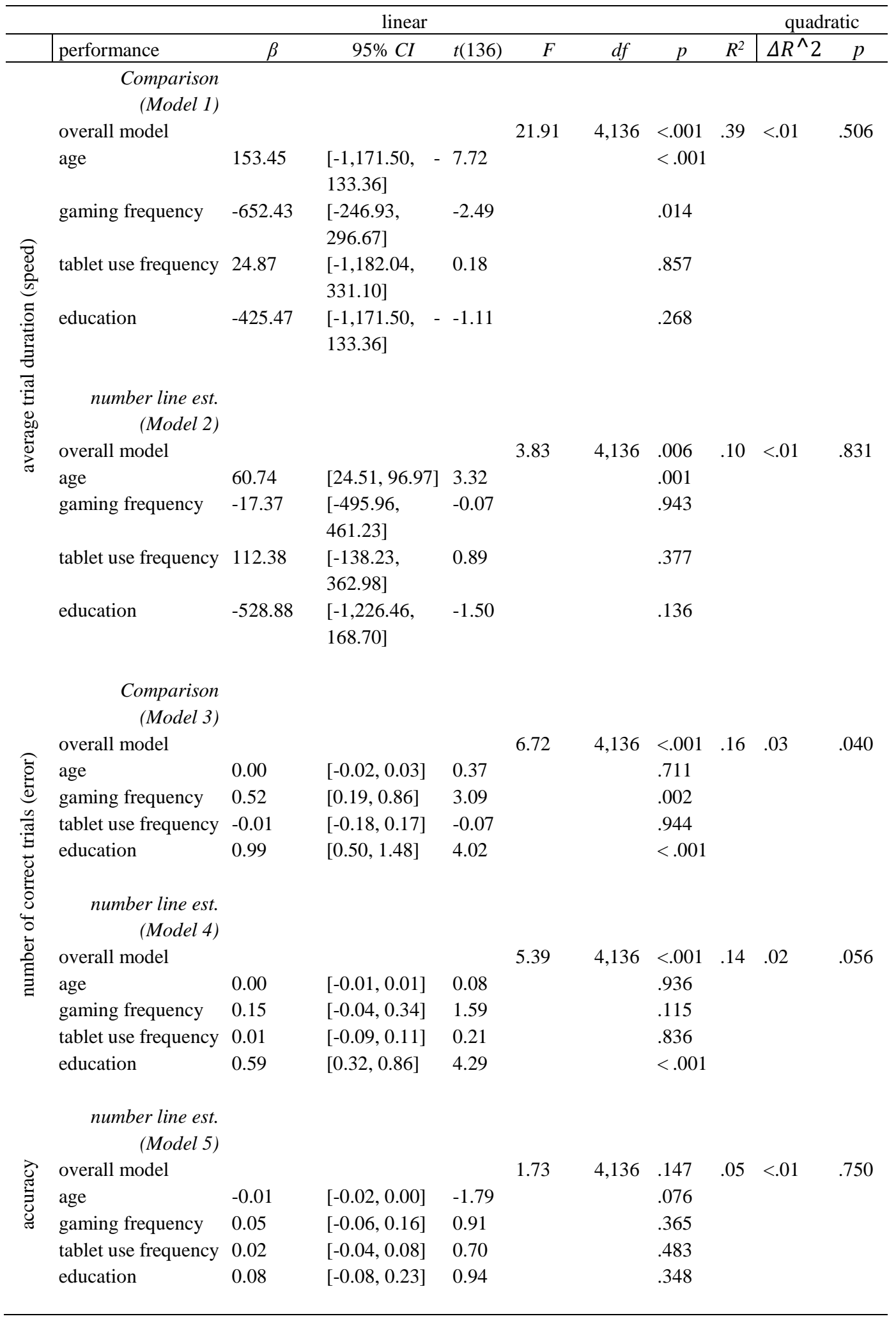


pag. 72

The third and fourth model are linked to crystallized aspects of intelligence. With the number of correct trials (e.g. error rates) as dependent variable in the comparison as well the number line estimation task were also significant. For the comparison task, gaming frequency and education predicted the number of errors made significantly. More frequent gamers as well as players with higher education achieved a higher number of correctly solved items and thus made fewer errors. For the estimation task, only education was a significant predictor with higher educated gamers making fewer errors.

Finally, with the fifth model (assuming combined aspects of intelligence) we could not accurately predict accuracy in the number line estimation tasks with our four predictors. Moreover, only one model significantly improved when modelling a quadratic age-related trend, namely the model on number of errors in the comparison task, i.e. the third model.

\subsection{Player experiences}

For the analyses of questionnaire data, it is more informative to look at differences at certain age levels rather than using age as a continuous variable. Thus, we used the separated three age groups to perform a multivariate analysis of variance (MANOVA) for each experiential questionnaire (Flow \& UEQ) considering the respective subscales as dependent variables. For the flow-subscales, the analysis revealed no significant main effect for age group [Pillai's trace $\left.=.02, F(2,137)=1.06, p=.348, \hat{\eta}_{\mathrm{G}}^{2}=.011\right]$. The same held true for the UEQsubscales [Pillai's trace $=.01, F(2,137)=0.44, p=.646, \hat{\eta}_{\mathrm{G}}^{2}=.005$; see Figure 4]. Therefore, no differences across age groups were identified on the domain of (subjective) player experience. Post-hoc tests revealed no detailed significant differences.

\subsection{Player attitudes}

Following the same procedure as before, the MANOVA results for the analysis of the computer literacy inventory (FIDEC) showed no main effect of age groups [Pillai's trace $=$ $.03, F(2,137)=2.07, p=.130, \hat{\eta}_{\mathrm{G}}^{2}=.003$ ]. Post-hoc tests showed age related differences between ratings for technology as useful tool for entertainment purposes. Both the younger and the middle-aged group showed higher ratings than the oldest group (both $p<.001$, fdrcorrected). Moreover, there was a marginally significant difference between the youngest and the oldest group with respect to negative valence (autonomous entity) for learning and work and societal consequences ( $p=.067$, fdr-corrected). No other significant differences between age groups were observed.

For the subscales of the technology usage inventory (TUI), we did not observe any statistically relevant group differences [Pillai's trace $=.01, F(2,135)=0.33, p=.718$, $\left.\hat{\eta}_{\mathrm{G}}^{2}=.003\right]$. Post-hoc tests indicated that merely the elderly group seemed to report marginally more general interest in technology than the youngest group $(\mathrm{p}=.063$, fdrcorrected).

Lastly, the analysis of the affinity for technology questionnaire (TA-EG) revealed a significant main effect for age group [Pillai's trace $=.10, F(2,135)=7.12 p=.001, \hat{\eta}_{\mathrm{G}}^{2}=.021$ ] In particular, post-hoc tests revealed that the elderly assumed significantly fewer negative consequences using electronic devices than the youngest group ( $p=.014, \mathrm{fdr}$-corrected). Attitudinal scales are depicted in Figure 5. 

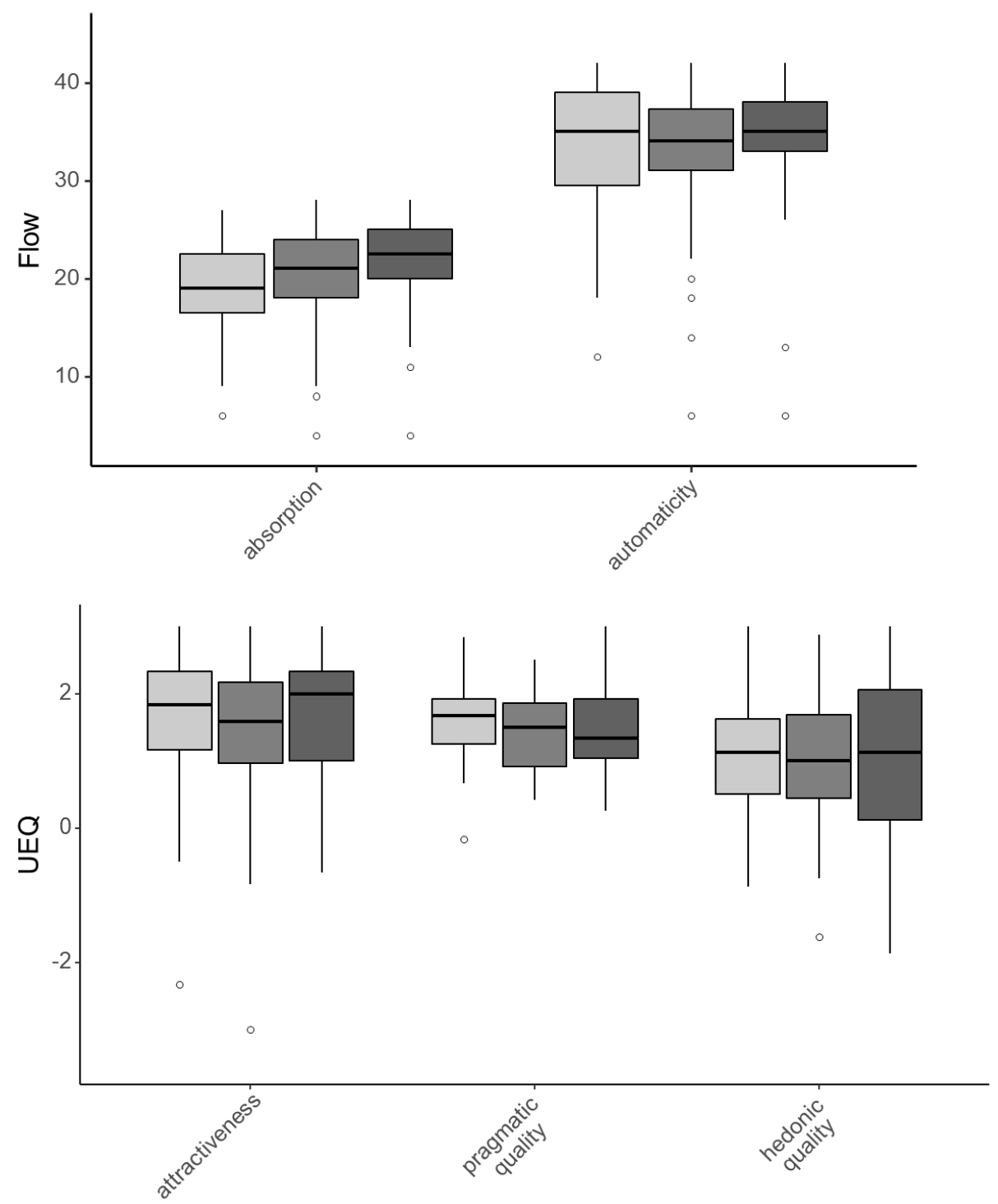

age

官 $<=34$

官 $35-59$

官 $=>60$

Figure 4. Questionnaire date for flow (upper panel) and general user experience (lower panel) during the interaction with the educational game across age groups. The bold horizontal line represents the median and the lower and upper hinges correspond to the first and third quartiles. Upper and lower whiskers extend from the hinge no further than $1.5 *$ inter-quartile range. Asterisks indicate significant comparisons. 

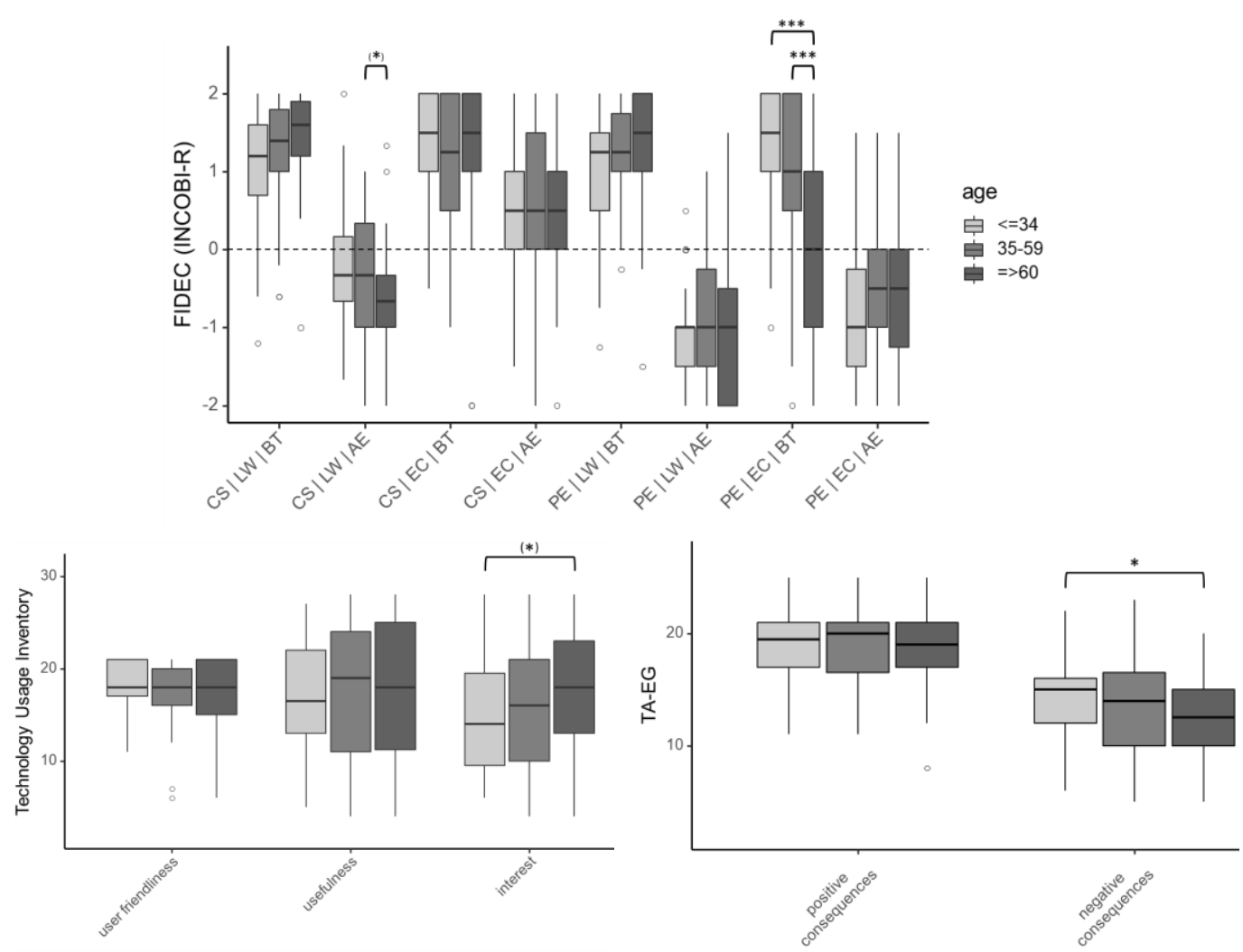

Figure 5. Player attitudes indicated by shortened versions of the computer literacy inventory (FIDEC, top chart; $C S=$ consequences for society; $P E=$ personal experience; $L W=$ learning \& work; $E C=$ entertainment \& communication; $B T=$ beneficial tool; $A E=$ autonomous entity), subscales from the technology usage inventory (TUI, bottom left panel) and the affinity for technology scales (TA-EG, bottom right panel). The bold horizontal line represents the median and the lower and upper hinges correspond to the first and third quartiles. Upper and lower whiskers extend from the hinge no further than $1.5 *$ inter-quartile range. Asterisks over brackets indicate significant comparisons.

\section{Discussion}

The current study aimed at shedding light on three domains of the applicability of educational games across age, (i) factors related to player cognition, such as performance (speed, errors, accuracy), (ii) subjective player experiences, such as flow experience, and a broader view on (iii) players' attitudes towards technology related to the device and in general. By investigating these domains in a sample from 19 to almost 80-year-old participants, we gained new insights into the applicability and usability of digital gamebased learning. Our results in terms of players' cognitions, as indicated by, for instance, ingame performance metrics, did show significant age differences predominantly in measures related to speed. In contrast, age groups did not differ on any of our player experience measures. Because the experiential ratings seem to overall be positive, there is no reasonable argument against a general applicability of our game. Even with respect to participants'/players' attitudes, significant differences were scarce. However, with one exception: older participants found technology for entertainment and communication purposes less useful to them personally than did younger participants. In the following, we will discuss our results on age effect on these three domains of game-based learning in more detail. 


\subsection{Player cognition}

The cognitive domain is of particular relevance when investigating digital game-based learning or educational games, respectively. Overall, our results are largely congruent with previous literature on cognitive ageing. We identified an overall, age-related decline in overall game performance which was composed of different in-game metrics. Importantly though, when differentiating overall game performance to reflect more specific in-game performance metrics (i.e., speed, error rates, and estimation accuracy) by considering relevant components of cognitive ageing (i.e., crystallized vs. fluid intelligence) a more diverse pattern of results emerged. As a strong presumption, speed related measures were expected to show a decline that should be mainly driven by age. In fact, this was the case for the number line estimation task as well as the comparison task. In particular, for the number line estimation task, age was the only and therefore main predictor of trial duration or speed, respectively. For the comparison task, not only age but also digital game experience was predictive of speed, respectively. That is, older participants and those with less experience with digital games responded slower in the comparison task. Apparently, the relationship between the two tasks and age was different.

For the comparison task, we might assume that on the one hand processing speed reflects an overall age-related performance decline, but, on the other hand, handling differences between tasks might have affected our results as well. These differences might have arisen because the comparison task required players to perform a more complex combination of tilting and pressing two additional buttons on the tablet to successfully solve an item as compared to the number line estimation task. More specifically, in magnitude comparison trials players had to pick up the stones/fractions (button press), place them by tilting the tablet according to their numerical magnitude, put the stones down, and, finally, press another button for confirming their response. This might have placed additional obstacles for older participants than the number line estimation task, where only tilting (i.e., navigating the avatar to the correct location) and one button press (i.e., confirming the location) was required.

However, this alternative interpretation was not substantiated by our results as player's tablet use did not significantly predict speed in magnitude comparison (or number line estimation). On the other hand, more regular gaming habits predicted faster task solving in the comparison task. This means more frequent players of digital games may handle, for instance, complex mechanical cascades with more ease due to more practice and experience.

From a cognitive ageing perspective, we argued that speed-related (in-game) metrics should primarily be affected by fluid components of intelligence, which is further substantiated by another result: The age of our sample started at 18, even before the alleged climax of any fluid performance decline. Yet, we could not observe a quadratic trend in our data, which may indeed be more indicative of an age-related decline in fluid components of intelligence. In this context, the fact that regular playing of digital games predicted speedperformance in comparison tasks is interesting, because this in turn implies an experiential component relevant for fast task completion in educational games. Lastly, we did find a quadratic relationship between age and speed in number-line estimation task.

Focusing on the number of errors participants made during task completion, we found that both errors in the number-line estimation task as well as the comparison task were not related to age, but they were related to participants' educational level. This is well in line with the assumption that the ability to locate fractions along a number line in adults relies on prior knowledge of number magnitudes and is therefore part of the crystallized, more stable domain of intelligence [53]. Therefore, the number of errors in both tasks were best explained by the educational background of the participants.

Interestingly, the number of errors seems to be the in-game metric in which a quadratic model of age tends to add up significantly in explaining the relationship. This leads to the presumption that we may be confronted with task relevant mental processes that rely more heavily on the interaction of fluid and crystallized parts of cognition. As described earlier, 
age in the comparison task was strongly related to speed, so fluid components of cognition seemingly play a major role. However, in the case of failing to solve the task correctly, crystallized knowledge about fractions and their magnitudes also came into play. In this case we may conclude that the quadratic relationship may indeed be indicative for the recruitment of more than just an incorrect knowledge representation.

Finally, accuracy in the number-line estimation task was neither predicted by age nor any other construct we addressed in this study. This can be interpreted in a way that when a player knows the answer, he/she is able to handle the game mechanics adequately, independent of age or, again, any other measure in our investigation. Unsurprisingly, there was also no non-linear relationship between age and accuracy in this task. This might be due to the fact that the ability to just correctly or accurately solve the tasks of the game is primarily knowledge-based. Because it is representing a successful recruitment of representational knowledge it is therefore part of the crystallized part of cognition which is assumed to be rather stable across age in adult individuals [27].

\subsection{Player experiences}

Apart from investigating players in-game performance across age, we also evaluated basic aspects of players' experiences with and around the game. In the current study we examined widely known and established constructs considered in game-based learning such as flow experience and general user experience. Both concepts showed similar effects across ages. In fact, no meaningful difference across age could be found on any of our employed scales or concepts, respectively. We may therefore assume, that participants in our sample experienced the game rather equally positive. Importantly, this also applies to the rating of the pragmatic quality of the game (e.g., controllability and handling). That is, even participants in the elderly group did not report any noticeable problems to accurately and smoothly operating the game. For most of them, the onboarding phase was more than enough to learn the mechanics the game required, and critical handling problems were virtually non-existent. At the same time apparently, some fraction estimations and comparisons were challenging for participants of all ages, so players were neither bored nor frustrated by the game. Accordingly, we found no signs of a decline of the perceived entertaining nature of the game. In sum, players across all age groups rated the game equally positive substantiating the applicability of educational games throughout the lifespan.

\subsection{Player attitudes}

Finally, we examined attitudinal differences regarding general computer literacy, attitudes towards the use of tablet-PCs and general attitudes towards technology. The first questionnaire FIDEC revealed that subjective ratings were more or less equally distributed regarding alleged consequences for society. In detail, across all age groups, participants found the use of technology for entertainment and communication as well in the learning and working context equally useful/useless when it comes to the society as a whole. The same held true for the subjective ratings with respect to personal experience in the learning and working context with no significant effect of age on either positive or negative ratings. There was only one exception to this in the entertainment \& communication ratings. The elderly group did not find technology as useful for entertainment and communication purposes on a personal level as the youngest and the middle-aged groups did. This may reflect that the older participants rely on other opportunities for personal entertainment than both younger groups and the use of technology for entertainment purposes may be less integral to their lives.

Furthermore, we found no age differences in the subjective ratings regarding the usefulness and user friendliness of using tablet-PCs.

Only the general interest in technology tended to vary across age with the elderly group showing marginally higher interest. The general perspective on technology, however, revealed that the elderly group did anticipate fewer negative consequences from the use of 
media and technology than the youngest group did. This is interesting in view of the assumption that the younger group has had different contact to technology from the beginning of their lives on. As mentioned before, the elderly group did not perceive computers as useful for personal entertainment in general. We initially assumed that some of the experience and handling aspects of our game would be related to the frequency by which our participants used such a device in general. This did not seem to be the case overall. Experience in using a tablet-PC had no explanatory power in any of our models.

Moreover, user-friendliness and usefulness of tablet-PCs were rated equally. In other words, a device-centred approach to evaluate our game was inconclusive. Nevertheless, we cannot exclude the possibility that these results might change with future generations or current generations that grew up with these technologies.

\subsection{Implications and future studies}

From a game-design perspective, we may conclude that games using, for instance, scaffolded feedback and high scores that depend on player's in-game performance should adapt these features to age appropriate norms. Especially with respect to speed related feedback elements, there might be the chance that, for example, inappropriate or even continuously lowered rewards because of lower speeded play might lead to undesirable effects like demotivation and frustration of elderly players.

The same seems to be reasonable for the actual knowledge content a learning game tries to convey. The present game requires the player to have accurate knowledge about fractions which may potentially be affected by a cognitive decline in terms of, for instance, rarely or even un-used knowledge. In this case, performance depends on the player's educational background and training of these representations. The resulting performance differences should also be considered by an adaptive game engine, as players may again get demotivated when they are confronted with failure too often. This might not have been that relevant in the current study as the learning session was rather short but could become a problem when longer and/or multiple learning sessions have to be completed in a training.

Future studies should continue to investigate factors influencing age-related performance differences in digital games. Our analyses revealed that age, gaming frequency, tablet use and educational level did not cover the entire variance at least in nonspeed-related in-game performance metrics of our study. Additionally, education/profession and gaming experience should be assessed and examined in more detail. Gaming experience may qualitatively vary over the lifespan in terms of, for instance, different game genres played or devices used by different age groups (e.g. [2], [20]). Among other things, current literature suggests that video game experience is differentially associated with cognitive markers like memory and attention in older adults compared to the younger population [20]. Additionally, more attention should be paid to the details of the relationship between experiential and performance domains in game-based learning environments across age. The particular learning domain (i.e. mathematics) as well as game design elements used might have had impact on our results. Caution should therefore be used when extrapolating effects to game-based learning in general. Our current study allows first conclusions regarding the relationship between age and other variables like cognition, experience or attitudes. However, those other variables were not put in relation to each other.

Another relevant aspect for future research is the demographic development within the population as briefly stated above. Currently, the older generation has not experienced technology while growing up the way the current or upcoming generations do or will do, for which digital gaming is probably a more integral or mainstream activity.

\subsection{Conclusion}

In the current work, we evaluated the age-related applicability of an educational game with an adult sample ranging from 19 to 79 years. Each participant played a tablet-based fraction 
pag. 78

learning game for $10-15$ minutes that consisted of two tasks, a number line estimation task and a comparison task. We covered relevant applicational aspects by assessing three indicative domains: player cognition (e.g. in-game performance metrics), player experiences (e.g. flow \& user experience) and player attitudes (e.g. attitudes towards technology/electronic devices).

In conclusion, we did only find an age-related decline in in-game performance metrics reflecting processing speed. On the other hand, educational background was strongly related to successfully solving the task and therefore relying on accurate fraction magnitude representations. However, the observed relationship was exclusively due to age in the number-line estimation task. In the comparison task, speed was also predicted by gaming frequency. Although designed for a different target group (i.e., elementary and secondary school students), we did not find any evidence that individuals of different, higher ages perceived or experienced the game differently, albeit their performance being fairly different. In sum, alongside literature-consistent performance differences, experiences with the math-game originally designed for a younger target group did not seem to vary significantly across adulthood. These results represent another piece of evidence that the pairing of playing and learning may be beneficial for everybody, at least independent of age. Furthermore, these results indicate that dissociating learning and playing in general and with advancements in the educational system has no pedagogical/didactical basis.

\section{References}

[1] A. Lenhart, J. Kahne, E. Middaugh, A. R. Macgill, C. Evans, and J. Vitak, 'Teens' gaming experiences are diverse and include significant social interaction and civic engagement', p. 76, 2008.

[2] ESA, 'Entertainment Software Association', Entertainment Software Association, May 02, 2019. https://www.theesa.com/wp-content/uploads/2019/05/ESA_Essential_facts_2019_final.pdf (accessed Jun. 24, 2019).

[3] J. Huizinga and A. Flitner, Homo Ludens: vom Ursprung der Kultur im Spiel, 25. Auflage. Hamburg: Rowohlt Taschenbuch Verlag, 2017.

[4] T. Hainey, T. M. Connolly, E. A. Boyle, A. Wilson, and A. Razak, 'A systematic literature review of games-based learning empirical evidence in primary education', Computers \& Education, vol. 102, pp. 202-223, Nov. 2016, doi: 10.1016/j.compedu.2016.09.001.

[5] C. P. Edwards, 'Three Approaches from Europe: Waldorf, Montessori, and Reggio Emilia.', Early Childhood Research \& Practice, vol. 4, no. 1, p. n1, 2002.

[6] M. Romero, H. Ouellet, and K. Sawchuk, 'Expanding the Game Design Play and Experience Framework for Game-Based Lifelong Learning (GD-LLL-PE)', in Game-Based Learning Across the Lifespan, M. Romero, K. Sawchuk, J. Blat, S. Sayago, and H. Ouellet, Eds. Cham: Springer International Publishing, 2017, pp. 1-11.

[7] U. Ritterfeld, M. J. Cody, and P. Vorderer, Eds., Serious games: mechanisms and effects. New York: Routledge, 2009.

[8] S. Greipl, K. Moeller, and M. Ninaus, 'Potential and Limits of Game-Based Learning', International Journal of Technology Enhanced Learning, in press.

[9] M. Ninaus, K. Kiili, J. McMullen, and K. Moeller, 'Assessing fraction knowledge by a digital game', Computers in Human Behavior, vol. 70, pp. 197-206, May 2017, doi: 10.1016/j.chb.2017.01.004.

[10] K. Kiili, K. Moeller, and M. Ninaus, 'Evaluating the effectiveness of a game-based rational number training - In-game metrics as learning indicators', Computers \& Education, vol. 120, pp. 13-28, May 2018, doi: 10.1016/j.compedu.2018.01.012.

[11] G. Gigerenzer, Calculated risks: how to know when numbers deceive you. New York: Simon \& Schuster, 2002.

[12] R. S. Siegler, L. K. Fazio, D. H. Bailey, and X. Zhou, 'Fractions: the new frontier for theories of numerical development', Trends in Cognitive Sciences, vol. 17, no. 1, pp. 13-19, Jan. 2013, doi: 10.1016/j.tics.2012.11.004.

[13] B. Bonnechère, B. Jansen, L. Omelina, and S. Van Sint Jan, 'The use of commercial video games in rehabilitation: a systematic review', International Journal of Rehabilitation Research, vol. 39, no. 4, pp. 277-290, Dec. 2016, doi: 10.1097/MRR.0000000000000190.

[14] H. Nguyen et al., 'Impact of Serious Games on Health and Well-being of Elderly: A Systematic Review', p. 10. 
[15] P. Rego, P. M. Moreira, and L. P. Reis, 'Serious games for rehabilitation: A survey and a classification towards a taxonomy', in 5th Iberian Conference on Information Systems and Technologies, Jun. 2010, pp. 1-6.

[16] F. Pallavicini, A. Ferrari, and F. Mantovani, 'Video Games for Well-Being: A Systematic Review on the Application of Computer Games for Cognitive and Emotional Training in the Adult Population', Front Psychol, vol. 9, Nov. 2018, doi: 10.3389/fpsyg.2018.02127.

[17] M. Romero, K. Sawchuk, J. Blat, S. Sayago, and H. Ouellet, Eds., Game-Based Learning Across the Lifespan: Cross-Generational and Age-Oriented Topics. Cham: Springer International Publishing, 2017.

[18] B. De Schutter, A. R. Roberts, and K. Franks, 'Miami Six-O: Lessons Learned From an Intergenerational Game Design Workshop', in Game-Based Learning Across the Lifespan, M. Romero, K. Sawchuk, J. Blat, S. Sayago, and H. Ouellet, Eds. Cham: Springer International Publishing, 2017, pp. 13-27.

[19] B. De Schutter and V. Vanden Abeele, 'Towards a Gerontoludic Manifesto', Anthropology \& Aging, vol. 36, no. 2, pp. 112-120, Nov. 2015, doi: 10.5195/aa.2015.104.

[20] H. Lee et al., 'Examining cognitive function across the lifespan using a mobile application', Computers in Human Behavior, vol. 28, no. 5, pp. 1934-1946, Sep. 2012, doi: 10.1016/j.chb.2012.05.013.

[21] B. Bonnechère, V. Sholukha, L. Omelina, M. Van Vooren, B. Jansen, and S. Van Sint Jan, 'Suitability of functional evaluation embedded in serious game rehabilitation exercises to assess motor development across lifespan', Gait \& Posture, vol. 57, pp. 35-39, Sep. 2017, doi: 10.1016/j.gaitpost.2017.05.025.

[22] C. Teulier, D. K. Lee, and B. D. Ulrich, 'Early gait development in human infants: Plasticity and clinical applications: Early Gait Development', Developmental Psychobiology, vol. 57, no. 4, pp. 447-458, May 2015, doi: 10.1002/dev.21291.

[23] K. M. Trewartha, A. Garcia, D. M. Wolpert, and J. R. Flanagan, 'Fast But Fleeting: Adaptive Motor Learning Processes Associated with Aging and Cognitive Decline', Journal of Neuroscience, vol. 34, no. 40, pp. 13411-13421, Oct. 2014, doi: 10.1523/JNEUROSCI.1489-14.2014.

[24] J. Wittland, P. Brauner, and M. Ziefle, 'Serious Games for Cognitive Training in Ambient Assisted Living Environments - A Technology Acceptance Perspective', in Human-Computer Interaction - INTERACT 2015, vol. 9296, J. Abascal, S. Barbosa, M. Fetter, T. Gross, P. Palanque, and M. Winckler, Eds. Cham: Springer International Publishing, 2015, pp. 453-471.

[25] A. D. Fisk, S. J. Czaja, W. A. Rogers, N. Charness, S. J. Czaja, and J. Sharit, Designing for Older Adults: Principles and Creative Human Factors Approaches, Second Edition, 0 ed. CRC Press, 2018.

[26] M. A. Farage, K. W. Miller, F. Ajayi, and D. Hutchins, 'Design Principles to Accommodate Older Adults', Global Journal of Health Science, vol. 4, no. 2, Feb. 2012, doi: 10.5539/gjhs.v4n2p2.

[27] J. C. Cavanaugh and F. Blanchard-Fields, Adult development and aging, 5th ed. Australia; Belmont, CA: Wadsworth/Thomson Learning, 2006.

[28] T. A. Salthouse, 'The Processing-Speed Theory of Adult Age Differences in Cognition', p. 26.

[29] F. I. M. Craik and E. Bialystok, 'Cognition through the lifespan: mechanisms of change', Trends in Cognitive Sciences, vol. 10, no. 3, pp. 131-138, Mar. 2006, doi: 10.1016/j.tics.2006.01.007.

[30] M. D. Arnoult, 'Ageing And Human Skill', Journal of Gerontology, vol. 14, no. 2, pp. 229-229, Apr. 1959, doi: 10.1093/geronj/14.2.229.

[31] R. Cooper et al., 'Age and Gender Differences in Physical Capability Levels from Mid-Life Onwards: The Harmonisation and Meta-Analysis of Data from Eight UK Cohort Studies', PLoS One, vol. 6, no. 11, Nov. 2011, doi: 10.1371/journal.pone.0027899.

[32] D. Finkel, M. Ernsth-Bravell, and N. L. Pedersen, 'Temporal Dynamics of Motor Functioning and Cognitive Aging', The Journals of Gerontology Series A: Biological Sciences and Medical Sciences, vol. 71, no. 1, pp. 109-116, Jan. 2016, doi: 10.1093/gerona/glv110.

[33] A. Barenbrock, M. Herrlich, and R. Malaka, 'Design lessons from mainstream motion-based games for exergames for older adults', in 2014 IEEE Games Media Entertainment, Oct. 2014, pp. 1-8, doi: 10.1109/GEM.2014.7048096.

[34] A. Perttula, K. Kiili, A. Lindstedt, and P. Tuomi, 'Flow experience in game based learning - a systematic literature review', International Journal of Serious Games, vol. 4, no. 1, Mar. 2017, doi: $10.17083 / i j s g . v 4 i 1.151$.

[35] K. Kiili, A. Lindstedt, and M. Ninaus, 'Exploring characteristics of students' emotions, flow and motivation in a math game competition', p. 10, 2018.

[36] M. Ninaus, K. Moeller, J. McMullen, and K. Kiili, 'Acceptance of Game-Based Learning and Intrinsic Motivation as Predictors for Learning Success and Flow Experience', International Journal of Serious Games, vol. 4, no. 3, Sep. 2017, doi: 10.17083/ijsg.v4i3.176.

[37] B. Laugwitz, M. Schrepp, and T. Held, 'Konstruktion eines Fragebogens zur Messung der User Experience von Softwareprodukten', in Mensch und Computer 2006: Mensch und Computer im Strukturwandel, München, 2006, pp. 125-134. 
pag. 80

[38] I. Giannopoulos, P. Kiefer, and M. Raubal, 'GazeNav: Gaze-Based Pedestrian Navigation', in Proceedings of the 17th International Conference on Human-Computer Interaction with Mobile Devices and Services - MobileHCl '15, Copenhagen, Denmark, 2015, pp. 337-346, doi: 10.1145/2785830.2785873.

[39] T. N. Friemel, 'The digital divide has grown old: Determinants of a digital divide among seniors', New Media \& Society, vol. 18, no. 2, pp. 313-331, Feb. 2016, doi: 10.1177/1461444814538648.

[40] S. E. Korupp, 'Causes and Trends of the Digital Divide', European Sociological Review, vol. 21, no. 4, pp. 409-422, Jun. 2005, doi: 10.1093/esr/jci030.

[41] A. Smith, 'Older Adults and Technology Use. Adomption is increasing, but many seniors remain insolated from digital life', 2018.

[42] S. J. Czaja and J. Sharit, 'Age Differences in Attitudes Toward Computers', J Gerontol B Psychol Sci Soc Sci, vol. 53B, no. 5, pp. P329-P340, Sep. 1998, doi: 10.1093/geronb/53B.5.P329.

[43] J. K. Eastman and R. Iyer, 'The impact of cognitive age on Internet use of the elderly: an introduction to the public policy implications', International Journal of Consumer Studies, vol. 29, no. 2, pp. 125-136, 2005, doi: 10.1111/j.1470-6431.2004.00424.x.

[44] J. Beddington and C. L. Cooper, 'The mental wealth of nations', vol. 455, p. 3, 2008.

[45] R. S. Siegler and J. E. Opfer, 'The development of numerical estimation: evidence for multiple representations of numerical quantity', Psychol Sci, vol. 14, no. 3, pp. 237-243, May 2003, doi: 10.1111/1467-9280.02438.

[46] D. C. Geary, M. K. Hoard, L. Nugent, and J. Byrd-Craven, 'Development of Number Line Representations in Children With Mathematical Learning Disability', Developmental Neuropsychology, vol. 33, no. 3, pp. 277-299, Apr. 2008, doi: 10.1080/87565640801982361.

[47] F. Rheinberg, R. Vollmeyer, and S. Engeser, 'Die erfassung des flow-erlebens', 2003.

[48] T. Richter, J. Naumann, and N. Groeben, 'Attitudes toward the computer: construct validation of an instrument with scales differentiated by content', Computers in Human Behavior, vol. 16, no. 5, pp. 473-491, Sep. 2000, doi: 10.1016/S0747-5632(00)00025-X.

[49] T. Richter, J. Naumann, and H. Horz, 'Eine revidierte Fassung des Inventars zur Computerbildung (INCOBI-R)', Zeitschrift für Pädagogische Psychologie, vol. 24, no. 1, pp. 2337, Jan. 2010, doi: 10.1024/1010-0652/a000002.

[50] O. D. Kothgassner, A. Felnhofer, N. Hauk, E. Kastenhofer, J. Gomm, and I. Kryspin-Exner, 'TUI', 2012.

[51] K. Karrer, C. Glaser, C. Clemens, and C. Bruder, 'Technikaffinität erfassen-der Fragebogen TAEG', Der Mensch im Mittelpunkt technischer Systeme, vol. 8, pp. 196-201, 2009.

[52] Y. Benjamini and Y. Hochberg, 'Controlling the false discovery rate: a practical and powerful approach to multiple testing', Journal of the Royal statistical society: series B (Methodological), vol. 57, no. 1, pp. 289-300, 1995.

[53] S. Trapp, S. Blömeke, and M. Ziegler, 'The openness-fluid-crystallized-intelligence (OFCl) model and the environmental enrichment hypothesis', Intelligence, vol. 73, pp. 30-40, Mar. 2019, doi: 10.1016/j.intell.2019.01.009. 